\title{
QUALIDADE DE VIDA PERCEBIDA \\ E ATIVIDADE FÍSICA: UM ESTUDO \\ EM IDOSAS ACIMA DE 80 ANOS \\ PARTICIPANTES DE UM PROGRAMA MUNICIPAL DE SAÚDE DA TERCEIRA IDADE NA SERRA GAÚCHA, RS
}

\author{
Neivo André Lima Bazzanella ${ }^{1}$ \\ João Carlos Jaccottet Piccoli² \\ Daniela Müller de Quevedo ${ }^{3}$
}

resumo

Trata-se de um estudo de caráter descritivo e de corte transversal que teve por objetivo verificar o nível de qualidade de vida percebida e de atividade física de idosas participantes do Programa Municipal de Saúde da Terceira Idade na Região da Serra Gaúcha, RS. Foram 58 idosas avaliadas, com idade igual ou superior a 80 anos. Os

\footnotetext{
1 Graduado em Enfermagem. Mestre em Diversidade Cultural e Inclusão Social. Enfermeiro Auditor Interno da Unimed Serra Gaúcha, RS. E-mail: neivobazzanella@bol.com.br

2 Graduado em Educação Física. Ph.D. em Educação Física, Ohio State University, Columbus, Ohio, USA. Professor titular da Universidade Feevale, RS, vinculado ao Curso de Educação Física e Programa de Pós-Graduação em Diversidade Cultural e Inclusão Social. E-mail: joaopiccoli@feevale.br

3 Graduada em Matemática. Doutora em Recursos Hídricos e Saneamento Ambiental. Professora adjunta à Universidade Feevale, RS, vinculada ao Curso de Administração e Programa de Pós-Graduação em Qualidade Ambiental. E-mail: danielama@feevale.br
} 
dados foram coletados entre outubro e novembro de 2013 através dos seguintes instrumentos de investigação: questionário sociodemográfico; WHOQOL-OLD, associado ao WHOQOL-BREF; e IPAQ, adaptado para idosos. Os resultados foram tabulados e submetidos para análise através de estatística descritiva e correlação de Spearman utilizando-se o SPSS versão 20.0. Os resultados do presente estudo apontam que 94,8\% das idosas possuem ensino fundamental incompleto, 82,8\% recebem de dois a cinco salários mínimos, 67,2\% residem sozinhas e 91,4\% são viúvas. Com uma pontuação de 60,25 土 8,25, as idosas têm uma percepção regular sobre a sua satisfação com a saúde e sua qualidade de vida e através do WHOQOL-Old: a maior pontuação foi observada nos domínios "Atividades passadas, presentes e futuras" (71,44 \pm 10,73) e "Participação social" (71,44 \pm 10,73), já a menor, no domínio "Intimidade" (23,81 \pm 30,35). Enquanto, pelo WHOQOL-Bref, as menores pontuações aconteceram no domínio físico, relativo a dor/desconforto, energia/fadiga, sono/repouso, mobilidade, atividades da vida cotidiana, dependência de mediação ou de tratamentos e/ou capacidade de trabalho $(65,20 \pm 9,93)$, as atividades físicas realizadas em diferentes domínios do IPAQ-Old, 100\% das idosas foram classificadas como insuficientemente ativas. Concluiu-se que foi constatada uma percepção satisfatória sobre a sua satisfação com a saúde e a qualidade de vida e uma classificação insuficientemente ativa na prática da atividade física.

palavras-chave

Qualidade de Vida. Atividade Física. Envelhecimento.

\section{Introdução}

O envelhecimento é um processo inevitável, dinâmico e associado a perdas tanto nos aspectos biológicos quanto nos aspectos socioafetivo e político, além de demandar vulnerabilidades que se diferenciam conforme gênero, idade, classe social, raça, local geográfico, entre outras variáveis. Essas vulnerabilidades impactam na expectativa de vida, na qualidade de vida e nas morbidade, mortalidade e incapacidades (ALENCAR; CARVALHO, 2009).

Conforme dados demográficos, há crescimento da população de idosos em países desenvolvidos e em desenvolvimento, como também o aumento do percentual de sujeitos com idade superior a 80 anos (ALENCAR; CARVALHO, 2009; IBGE, 2012). Este fato tem proporcionado algumas reflexões e discussões, 
pois o envelhecimento populacional provoca modificações físicas, biológicas, psicológicas e sociais no perfil do indivíduo. O idoso na faixa etária acima dos 80 anos apresenta algumas especificidades, ainda não bem caracterizadas, relacionadas às suas fragilidades físicas e cognitivas, à sua funcionalidade $\mathrm{e}$ ao seu maior grau de dependência (XAVIER et al., 2010).

Essas alterações e desgastes em vários sistemas funcionais, que são progressivas e irreversíveis, diferenciam-se de um indivíduo para outro. Entretanto, nas idades mais avançadas, as limitações visuais, auditivas, motoras e intelectuais, bem como o surgimento de doenças crônico-degenerativas, intensifica-se, interferindo na capacidade funcional e ocasionando a dependência nas atividades cotidianas e comprometendo a qualidade de vida do idoso (DOMINGUES; NERI, 2009; XAVIER et al., 2010).

A capacidade funcional pode ser definida como o potencial que os idosos apresentam para decidir e atuar em suas vidas independentemente, no seu cotidiano, de forma segura, eficaz e com um gasto mínimo de energia (MATSUDO, 2000). O declínio da capacidade funcional, que aumenta com a idade, está associado a fatores clínicos, estes ligados à capacidade para desempenhar as atividades de vida diária e participar socialmente, mediado pelo contexto ambiental e pessoal. A doença participa dessa rede causal, mas sua presença não está necessariamente relacionada com a perda da autonomia. A capacidade funcional surge, então, como novo conceito em saúde, sendo relevante em Gerontologia como indicativo de qualidade de vida (ARAÚJO; CEOLIM, 2007).

Entender o processo do envelhecimento humano associado à qualidade de vida assume múltiplas definições. É um processo natural caracterizado por um declínio das funções, pois envolve modificações biológicas, psicológicas que diferenciam a sabedoria, a emoção e as modificações sociais que humanizam o homem. A compreensão de qualidade de vida na velhice adquire aspectos específicos e é nessa fase da vida que as diferenças individuais se intensificam mais do que em qualquer outra faixa etária (ALEXANDRE; CORDEIRO; RAMOS, 2009; OLIVEIRA et al., 2010).

Para o idoso, a qualidade de vida está associada ao bem-estar como resultado do que a pessoa realizou e a sua satisfação em relação àquilo que idealizava como essencial para a sua vida. Contudo, ela está atrelada à habilidade de o idoso adaptar suas dimensões cognitivas, comportamental, emocional, social e em manter sua independência e autonomia, o que deve ser planejado ao longo de sua vida (OLIVEIRA et al., 2010). O envelhecimento bem-sucedido passa a ser o resultado da interação de fatores multidimensionais, que incluem questões relacionadas à saúde física e mental, independência na vida diária, aspectos econômicos e psicossociais (SANTOS et al., 2007). 
Ao processo de envelhecimento, estão associadas diversas alterações que causam dano aos diferentes sistemas do organismo. Numa das principais alterações que surgem com o avanço cronológico, está o decréscimo da função muscular, interferindo diretamente a capacidade de realizar tarefas cotidianas, diminuindo a independência funcional e, desse modo, refletindo negativamente na qualidade de vida do idoso. Os dados obtidos através da revisão de literatura, realizada por Lacourt e Marini (2006), com o objetivo de revisar os principais fatores envolvidos com o prejuízo da função muscular associado ao envelhecimento e a sua influência na qualidade de vida dos idosos, apontam que tanto a força muscular quanto a resistência e a potência sofrem significativa redução com o avanço da idade e que este prejuízo da função muscular pode ser considerado um dos principais fatores que interferem na qualidade de vida do idoso (LACOURT; MARINI, 2006).

A capacidade funcional é um dos fatores para avaliar a saúde do idoso, que é uma resultante entre saúde física, mental, independência na vida diária, integração social, suporte familiar, independência econômica e utilização de serviços. Frequentemente, essa avaliação se dá pela capacidade de realizar as atividades da vida diária (AVD) (AIRES; PASKULIN; MORAIS, 2010).

A partir dessa contextualização, idealizou-se a presente investigação que teve, por objetivo, verificar o nível de qualidade de vida percebida e de atividade física de idosas acima de 80 anos participantes do Programa Municipal de Saúde da Terceira Idade na Serra Gaúcha, RS.

\section{Materiais e métodos}

A presente investigação possui caráter descritivo e de corte transversal. A amostra do estudo foi composta por 58 idosas selecionadas através de uma amostragem não probabilística e, por conveniência, com idade igual e superior a 80 anos, participantes do Programa de Saúde para Adulto e Terceira Idade ${ }^{4}$ da Secretaria Municipal do Esporte e Lazer de Caxias do Sul -SMEL, Prefeitura de Caxias do Sul, RS. Às selecionadas, o pesquisador apresentou a sua proposta do

4 O Programa de Saúde para Adulto e Terceira Idade da SMEL de Caxias do Sul é composto por 72 grupos de atividades realizadas uma vez por semana, com duração de 90 minutos, em locais disponibilizados pela comunidade, tais como: Centros Comunitários, salões paroquiais, ginásios, clubes, dentre outros. O Projeto oportuniza à comunidade urbana e rural do município atividades de participação contínua elaboradas, organizadas e orientadas por profissionais da área de Educação Física, enfatizando, em seus atendimentos, os aspectos socioafetivos, físicos, biológicos e espirituais. Os participantes escolhem os turnos desejados, se matutino ou tarde, e nos locais, são propostas atividades de convívio social, de lazer, culturais e educacionais, além de atividades físicas e de relaxamento. 
estudo, quando assinaram o Termo de Consentimento Livre e Esclarecido, e foram agendados hora e local de quando e onde foram realizadas as coletas de dados.

Foram excluídas do estudo aquelas que se recusaram a assinar o Termo de Consentimento Livre e Esclarecido, não aceitando participar da coleta de dados ou não comparecendo nos dias de coletas de dados.

A coleta de dados ocorreu no período de outubro a novembro de 2013 pelo pesquisador, pelo orientador e por um grupo de cinco alunos, devidamente treinados pelo segundo para a aplicação dos instrumentos propostos. Foram avaliadas, em média, 10 idosas em cada dia de coleta, sendo que as atividades ocorreram nos dois períodos, manhã e tarde. Os dados foram coletados em salas cedidas pelo Centro de Saúde da Faculdade da Serra Gaúcha - FSG, em Caxias do Sul, RS.

Quanto aos aspectos éticos, conforme Resolução № 466/2012, do Conselho Nacional de Saúde em Pesquisa, envolvendo seres humanos, este projeto foi encaminhado para apreciação do Comitê de Ética em Pesquisa da Universidade FEEVALE e aprovado, CAE no $\mathbf{1}$ 196622137.0000.5348.

Para a coleta de dados, foram utilizados os seguintes instrumentos:

a) Questionário sociodemográfico com o objetivo de conhecer o perfil e descrever as características sociodemográficas da amostra como, sexo, idade, data de nascimento, escolaridade, renda familiar, aposentadoria, condições da moradia e se havia convívio com outras pessoas na residência;

b) Questionário de Avaliação da Qualidade de Vida da Organização Mundial de Saúde - WHOQOL - OLD, composto por dimensões positivas e negativas, totalizando 24 itens atribuídos em seis domínios: Domínio 1 Funcionamento do sensório (FS), questões 1, 2, 10 e 20; Domínio 2 - Autonomia (AUT), questões 3, 4, 5 e 11; Domínio 3 - Atividades passadas, presentes e futuras (PPF), questões 12, 13, 15 e 19; Domínio 4 - Participação social (PSO), questões 14, 16, 17 e 18; Domínio 5-Morte e morrer (MEM), questões 6, 7, 8 e 9 e Domínio 6 - Intimidade (INT), questões 21, 22, 23 e 24. Cada um dos domínios possuía quatro itens; portanto, para todos os domínios, o escore dos valores possíveis poderia oscilar de 4 a 20 pontos desde que todos os itens de um domínio fossem respondidos (FLECK; CHACHAMOVICH; TRENTIN, 2006).

Os escores destes seis domínios ou os valores dos 24 itens do módulo WHOQOL-OLD podem ser combinados para produzir um escore geral ("global") para a qualidade de vida em adultos idosos, denotado como o "escore total" do módulo WHOQOL-OLD. O instrumento não possui um ponto de corte, entretanto, quanto mais alto for o escore, melhor é a Qualidade de Vida da pessoa que o respondeu. O questionário WHOQOL-OLD foi aplicado juntamente ao questionário WHOQOL-BREF por orientação dos autores; 
c) WHOQOL-BREF, forma reduzida de avaliação da qualidade de vida, questionário composto por 26 questões, dividido em quatro domínios. As duas primeiras perguntas iniciais do instrumento questionam sobre a qualidade de vida geral e a saúde, respectivamente, e as demais 24 , intituladas de facetas, estão distribuídas nos domínios: Físico, Psicológico, Relações Sociais e Meio Ambiente. As respostas seguem uma escala de Likert, de 1 a 5, isto é, quanto maior a pontuação, melhor a qualidade de vida;

d) Questionário Internacional de Atividade Física - IPAQ, adaptado para idosos por Mazo e Benedetti (2010), permite estimar o dispêndio energético semanal de atividades físicas relacionadas com o trabalho, transporte, tarefas domésticas e lazer realizadas por, pelo menos, 10 minutos contínuos, com intensidade moderada e vigorosa, durante uma semana normal/habitual.

O instrumento em questão foi desenvolvido a partir das dificuldades encontradas para se obter medidas de atividades físicas internacionalmente comparáveis. Quanto à sua aplicação em população idosa, algumas dificuldades foram apresentadas pelas idosas quanto à mensuração da quantidade de dias (semana normal/habitual), do tempo (horas e minutos por dia e semana) e da intensidade (leve ou moderada ou vigorosa) na realização das atividades físicas nos diferentes domínios do IPAQ (trabalho, transporte, tarefas domésticas, lazer e tempo sentado) (MAZO; BENEDETTI, 2010).

O IPAQ adaptado para idosos é um questionário subdividido em cinco domínios (Atividades físicas no trabalho; Atividades físicas como meio de transporte; Atividades físicas em casa: tarefas domésticas e família; Atividades físicas de recreação, esporte, exercício físico e de lazer; e Tempo gasto sentado). Cada domínio é composto por 4, 3, 3, 3 e 2 questões, respectivamente, totalizando 15 questões (MAZO; BENEDETTI, 2009).

Suas informações permitem estimar o tempo despendido por semana em diferentes dimensões de atividade física (caminhadas e esforços físicos de intensidades moderada e vigorosa) e de inatividade física (posição sentada), com os resultados transformados em METs (equivalente metabólico), quantidade de energia gasta por um corpo enquanto em repouso.

A partir desse estado, os METs são aumentados na medida em que a intensidade da atividade for, também, aumentada. A unidade de medida MET-min por semana equivale ao nível de MET relacionado à atividade (caminhada, atividades moderadas e vigorosas) multiplicado pelo tempo gasto em minutos e pela frequência semanal de prática da atividade física. Assim, a caminhada equivale a 3,3 METs, as atividades moderadas a 4,0 METs e as atividades vigorosas a 8,0 METs, com os METs sendo multiplicados pelo tempo gasto e pela frequência semanal de prática. 
Para a classificação dos sujeitos nos níveis de atividade física, utilizaram-se as categorias oficiais do IPAQ: Insuficientemente ativos - aqueles sujeitos que não se enquadrassem em nenhuma das categorias que se seguem ou que não praticassem atividades físicas; Suficientemente ativos - aqueles sujeitos que praticassem 5 ou mais dias de qualquer combinação de caminhada, atividades moderadas ou vigorosas, alcançando o mínimo de 600 MET-min/sem e Muito ativos - aqueles sujeitos que praticassem no mínimo 3 dias de atividades vigorosas e acumulassem um mínimo de 1500 MET-min/semana ou 7 dias de qualquer combinação de caminhada, atividade moderada ou vigorosa, alcançando no mínimo 3.000 METs-min/semana.

Os resultados foram tabulados e submetidos a análise através de estatística descritiva e cálculo de frequências absolutas e relativas dos casos observados. Os dados foram, primeiramente, submetidos ao teste de normalidade de Kolmogorov-Smirnov $(\alpha=0,05)$ para se verificar a normalidade dos dados. Foi, então, utilizada a Correlação de Spearman $(\alpha=0,05)$ para se associarem as questões gerais e domínios do WHOQOL-BREF. Os dados foram submetidos ao pacote estatístico SPSS versão 20.0.

\section{Resultados}

O estudo apontou que a amostra investigada era composta exclusivamente por mulheres.

Tabela 1 - Distribuição das frequências absolutas e relativas das características sociodemográficas das idosas participantes de grupos de convivência de Caxias do Sul, RS ( $n=58$ ).

\begin{tabular}{l|l|c|c}
\hline \multicolumn{1}{c|}{ Variável } & \multicolumn{1}{c|}{ Categorias } & f & $\%$ \\
\hline \multirow{4}{*}{ Escolarização } & Ensino fundamental incompleto & 55 & 94,8 \\
\cline { 2 - 4 } & Ensino fundamental completo & 1 & 1,7 \\
\cline { 2 - 4 } & Ensino médio completo & 2 & 3,4 \\
\hline \multirow{3}{*}{ Renda } & 1 salário mínimo & 8 & 13,8 \\
\cline { 2 - 4 } & 2 a 5 salários mínimos & 48 & 82,8 \\
\cline { 2 - 4 } & 6 a 9 salários mínimos & 2 & 3,4 \\
\hline \multirow{2}{*}{ Situação funcional } & Aposentada & 6 & 89,7 \\
\cline { 2 - 4 } & Não aposentada & & 3,4 \\
\hline
\end{tabular}




\begin{tabular}{l|l|c|c}
\hline \multicolumn{1}{c|}{ Varininuáắa } & \multicolumn{1}{|c|}{ Categorias } & $\mathbf{f}$ & $\%$ \\
\hline \multirow{4}{*}{ Residência } & Sozinha & 39 & 67,2 \\
\cline { 2 - 4 } & Com filho(s) & 11 & 19,0 \\
\cline { 2 - 4 } & Com companheiro & 5 & 8,6 \\
\cline { 2 - 4 } & Própria & 57 & 98,3 \\
\cline { 2 - 4 } & Alugada & 1 & 1,7 \\
\hline \multirow{2}{*}{ Estado civil } & Casada/morando junto & 5 & 8,6 \\
\cline { 2 - 4 } & Viúva & 53 & 91,4 \\
\hline
\end{tabular}

O perfil da amostra, constatado na Tabela 1, denota que a maioria das idosas avaliadas se encontrava na faixa etária de 80 a 89 anos, com apenas uma idosa acima de 90 anos; idade média de 82,4 anos e desvio padrão de 2,4 anos; todas eram alfabetizadas e $94,8 \%$ (55) possuíam o ensino fundamental incompleto; 89,7\% (52) eram aposentadas; 82,4\% (48) tinham uma renda mensal que variava entre dois a cinco salários mínimos; $67,2 \%$ (39) moravam sozinhas; e 98,3\% (57) possuíam casa própria e a maioria era viúva.

Tabela 2 - Distribuição dos escores médios e desvios-padrão (DP) dos escores originais e transformados dos resultados separados por domínio avaliado do WHOQOL-OLD e WHOQOL-BREF $(n=58)$.

\begin{tabular}{c|l|c|c|c}
\hline \multirow{4}{*}{ Instrumentos } & \multicolumn{1}{|c|}{ Domínios } & $\begin{array}{c}\text { Escores } \\
\mathbf{( 1 - 5 )}\end{array}$ & \multicolumn{2}{c}{$\begin{array}{c}\text { Escores } \\
\text { Transformados }\end{array}$} \\
\cline { 3 - 5 } & & Médias & Médias & \multicolumn{1}{c}{ DP } \\
\hline \multirow{4}{*}{ WHOQOL-OLD } & Funcionamento dos sentidos & 3,40 & 60,23 & 13,93 \\
\cline { 2 - 5 } & Autonomia & 3,75 & 68,85 & 11,31 \\
\cline { 2 - 5 } & Atividades passadas, presentes e futuras & 3,85 & 71,44 & 10,73 \\
\cline { 2 - 5 } & Participação social & 3,85 & 71,44 & 10,73 \\
\cline { 2 - 5 } & Morte e morrer & 3,62 & 65,73 & 25,14 \\
\cline { 2 - 5 } & Intimidade & 1,95 & 23,81 & 30,35 \\
\cline { 2 - 5 } & Escore total & 3,41 & 60,25 & 8,25 \\
\hline \multirow{5}{*}{ WHOQOL-BREF } & Físico & 14,43 & 65,20 & 9,93 \\
\cline { 2 - 5 } & Psicológico & 14,60 & 66,30 & 8,19 \\
\cline { 2 - 5 } & Relações sociais & 15,17 & 69,82 & 7,61 \\
\cline { 2 - 5 } & Meio ambiente & 14,92 & 68,26 & 8,00 \\
\cline { 2 - 5 } & Questão geral 1 & - & 71,55 & 12,78 \\
\cline { 2 - 5 } & Questão geral 2 & & 65,51 & 17,41 \\
\hline
\end{tabular}

A Tabela 2 revela que o escore médio total de qualidade de vida percebida pelas idosas através do WHOQOl-OLD foi de $60,25 \pm 8,25$ pontos de uma escala transformada de 1-100, em que a maior pontuação (escore) caracteriza como 
uma melhor qualidade de vida e, a menor, uma pior qualidade de vida. A maior pontuação foi observada nos domínios "Atividades passadas, presentes e futuras" (71,44 $\pm 10,73)$ e "Participação social" $(71,44 \pm 10,73)$ e, a menor, no domínio "Intimidade" $(23,81 \pm 30,35)$.

Na mesma tabela, também, observam-se as pontuações obtidas pela amostra no WHOQOL-BREF, instrumento aplicado juntamente ao WHOQOL-OLD, em que o escore médio de avaliação da percepção da qualidade de vida geral da amostra do estudo encontra-se em 71,55 $\pm 12,78$. As menores pontuações aconteceram no domínio "físico", relativo a dor/desconforto, energia/ fadiga, sono/repouso, mobilidade, atividades da vida cotidiana, dependência de mediação ou de tratamentos e/ou capacidade de trabalho $(65,20 \pm 9,93)$ e na questão geral sobre a satisfação com saúde, cuja pontuação foi $65,51 \pm 17,41$.

Tabela 3 - Nível de correlação e significância das variáveis avaliações da qualidade de vida geral (Q.1) e satisfação com a saúde (Q.2) do WHOQOL-BREF aplicado na amostra $(n=58)$.

\begin{tabular}{|c|c|c|c|c|}
\hline \multirow{2}{*}{$\begin{array}{c}\text { Domínios } \\
\text { WHOQOL-BREF }\end{array}$} & \multicolumn{2}{|c|}{ Avaliação da QV geral } & \multicolumn{2}{|c|}{ Satisfação com a saúde } \\
\hline & Rho( $\rho)$ & $p$ & Rho( $\rho)$ & $p$ \\
\hline Físico & $0,591^{\star \star}$ & 0,000 & $0,659^{\star \star}$ & 0,000 \\
\hline Psicológico & $0,304^{\star}$ & 0,020 & $0,333^{*}$ & 0,011 \\
\hline Relações sociais & $-0,003$ & 0,983 & 0,109 & 0,414 \\
\hline Meio ambiente & $0,513^{\star \star}$ & 0,000 & $0,447^{\star \star}$ & 0,000 \\
\hline
\end{tabular}

A Tabela 3 apresenta os coeficientes de correlação de Spearman e significâncias entre as variáveis "avaliação da qualidade de vida geral" e "satisfação com a saúde" do WHOQOL-BREF associados aos domínios do instrumento. Constata-se, nesta tabela, que a percepção da amostra, ao avaliar a sua qualidade de vida geral e saúde, está associada, principalmente, aos domínios "físico" (dor e desconforto, energia e fadiga, sono e repouso, mobilidade, atividades da vida cotidiana, dependência de medicação ou de tratamentos e capacidade para o trabalho) e "meio ambiente" (segurança física e proteção, ambiente no lar, recursos financeiros, cuidados de saúde e sociais, oportunidades de adquirir novas informações e habilidades, participação em oportunidades de recreação/lazer, ambiente físico e transporte).

No domínio "físico", foi constatada uma correlação positiva forte muito significativa em relação à "satisfação com a saúde" ( $(\mathrm{Q}=0,659, p \leq 0,01)$ e à "avaliação da qualidade de vida geral", uma correlação positiva moderada ( $(=0,591, p \leq 0,01)$. Assim, à medida que a amostra percebia melhor sua qualidade de vida geral e satisfação com a saúde, assim o fazia no domínio "físico" ou vice-versa. 
No domínio "meio ambiente", foi observada uma correlação positiva moderada muito significativa em relação às "satisfação com a saúde" ( $\varrho=0,447$, $p \leq 0,01)$ e "avaliação da qualidade de vida geral" ( $(\mathrm{Q}=0,513, p \leq 0,01)$. Tais dados levam à ideia de que as idosas avaliadas que possuíam uma percepção satisfatória de qualidade de vida geral e de satisfação com a saúde, também, a possuíam no domínio "meio ambiente" ou vice-versa.

Em relação ao domínio "psicológico" (sentimentos positivos; pensar, aprender, memória e concentração; autoestima; imagem corporal e aparência; sentimentos negativos e espiritualidade, religião e crenças pessoais) associado às "avaliação da qualidade de vida geral" e "satisfação com a saúde", foi verificada uma correlação positiva moderada significativa, isto é, $\mathrm{Q}=0,304, p \leq 0,05 \mathrm{e}$ $\varrho=0,333, p \leq 0,05$, respectivamente. Este fato indicou, também, que uma percepção satisfatória de qualidade de vida geral e de satisfação com a saúde, também, acontecia no domínio "psicológico" da amostra do estudo ou vice-versa.

No domínio "relações sociais" associado às questões gerais do WHOQOL-BREF, não apresentou associação estatística significativa $(p \geq 0,05)$. Assim, uma percepção satisfatória de qualidade de vida geral e de satisfação com a saúde, não estavam associadas à constatada no domínio "relações sociais" (relações pessoais, suporte social e atividade sexual) pela amostra.

Tabela 4 - Distribuição das frequências absolutas e relativas da amostra separada por classificação do IPAQ adaptado a idosos ( $n=58)$.

\begin{tabular}{l|c|c}
\multicolumn{1}{c|}{ Classificação } & f & \% \\
\hline Muito ativo & 1 & 1,7 \\
\hline Suficientemente ativo & 11 & 19,0 \\
\hline Insuficientemente ativo & 46 & 79,3 \\
\hline
\end{tabular}

Na Tabela 4, considerando-se as atividades físicas realizadas em diferentes domínios (trabalho, transporte, atividades domésticas e lazer) do IPAQ adaptado pela amostra do estudo, 79,3\% das idosas foram classificadas como insuficientemente ativas, isto é, não atingiram um mínimo de 5 ou mais dias de qualquer combinação de caminhada, atividades moderadas ou vigorosas, alcançando um mínimo de 600 MET-min/sem, classificação esta suficientemente ativa. 
Tabela 5 - Distribuição das médias e desvios-padrão do tempo em minutos, frequência e METs semanais das atividades físicas realizadas, separadas por domínios do IPAQ adaptado a idosos $(n=58)$

\begin{tabular}{|c|c|c|c|c|c|c|c|}
\hline \multirow{2}{*}{ Domínios } & \multirow{2}{*}{ Atividades } & \multicolumn{2}{|c|}{$\begin{array}{l}\text { Tempo (minutos } \\
\text { semanais) }\end{array}$} & \multicolumn{2}{|c|}{$\begin{array}{c}\text { Frequência } \\
\text { semanal (dias) }\end{array}$} & \multicolumn{2}{|c|}{ METs semanais } \\
\hline & & Média & $\begin{array}{l}\text { Desvio } \\
\text { padrão }\end{array}$ & Média & $\begin{array}{l}\text { Desvio } \\
\text { padrão }\end{array}$ & Média & $\begin{array}{l}\text { Desvio } \\
\text { padrão }\end{array}$ \\
\hline $\begin{array}{l}\text { Atividade } \\
\text { física como } \\
\text { meio de } \\
\text { transporte }\end{array}$ & Caminhada & 87,5 & 83,6 & 3,1 & 1,9 & 1199,6 & 1516,9 \\
\hline \multirow{2}{*}{$\begin{array}{l}\text { Atividade } \\
\text { física em } \\
\text { casa ou } \\
\text { apartamento: } \\
\text { trabalho, } \\
\text { tarefas } \\
\text { domésticas } \\
\text { e cuidar da } \\
\text { família }\end{array}$} & $\begin{array}{l}\text { Atividades } \\
\text { moderadas } \\
\text { ao redor } \\
\text { da casa ou } \\
\text { apartamento }\end{array}$ & 121,2 & 207,7 & 2,4 & 2,1 & 1926,2 & 3332,4 \\
\hline & $\begin{array}{l}\text { Atividade } \\
\text { física mode- } \\
\text { rada dentro } \\
\text { da casa ou } \\
\text { apartamento }\end{array}$ & 242,5 & 264,8 & 3,9 & 2,1 & 3610,6 & 5055,1 \\
\hline \multirow{2}{*}{$\begin{array}{l}\text { Atividades } \\
\text { físicas de } \\
\text { recreação, } \\
\text { esporte, } \\
\text { exercício } \\
\text { e de lazer }\end{array}$} & Caminhada & 39,8 & 63,0 & 0,83 & 1,1 & 319,7 & 724,0 \\
\hline & $\begin{array}{l}\text { Atividades } \\
\text { moderadas } \\
\text { no tempo } \\
\text { livre }\end{array}$ & 113,7 & 47,9 & 1,4 & 0,6 & 740,6 & 660,7 \\
\hline
\end{tabular}

Analisando-se separadamente os resultados apresentados na Tabela 5, constata-se que as idosas avaliadas praticavam numa média de $242,5 \mathrm{~min} /$ semanais de atividades físicas moderadas dentro da casa ou apartamento que significa uma média diária de 34 minutos de atividades.

Tomando-se como referência o envolvimento da amostra em atividades físicas de recreação, esporte, exercício e de lazer, observa-se que isto ocorre em média um dia por semana com duração de 21 minutos.

Constatou-se, então, um baixo envolvimento das idosas avaliadas na prática de atividades físicas, concentrando-se, tal prática, nas lidas domésticas, em casa ou no apartamento. Assim, a amostra carece de um maior envolvimento na prática de atividades físicas de recreação, esporte, exercício e lazer para a melhoria dos padrões de saúde e de qualidade de vida. 
Os resultados do presente estudo não evidenciaram associação estatística significante para as idosas participantes em grupos de convivência associada com a qualidade de vida e com o nível de atividade física.

Pelo fato de apenas um idoso participante do Programa de Saúde para Adulto e Terceira Idade da Secretaria Municipal do Esporte e Lazer de Caxias do Sul-SMEL, Prefeitura de Caxias do Sul, RS ter se disponibilizado a participar e, considerando-se que do total de 146 idosos, 93,83\% são mulheres e apenas 6,17\% de homens participam desse Programa, a participação feminina nos grupos de convivência é superior à participação masculina, fenômeno este que, possivelmente, tenha sido consequência no aumento da expectativa de vida e da longevidade ser maior nas mulheres do que nos homens (CAXIAS DO SUL, 2012). Dessa forma, acarreta um número expressivo de viúvas que participam nos grupos de convivência (BENEDETTI; MAZO; BORGES, 2012). Isso pode ser observado neste estudo uma vez que do total de mulheres ( $\mathrm{n}=58)$, apenas $8,6 \%$ das idosas vivem com o companheiro, enquanto, das demais, $91,4 \%$ eram viúvas.

A predominância das mulheres nos grupos de convivência é um fato, também, observado em vários dos referenciais citados neste estudo, haja vista a feminização do envelhecimento (BORGES et al., 2008; BRAGA et al., 2011; SERBIM; FIGUEIREDO, 2011; LEITE et al., 2012). No entanto, os estudos citados pesquisaram idosos na faixa etária acima de 60 anos, enquanto o presente estudo delimitou a faixa etária de 80 anos ou mais. Entre as idosas desse estudo, é verificada a baixa escolaridade: $94,8 \%$ até o quarto ano do ensino fundamental e, na sua maioria, são viúvas, resultado que também se evidenciou em outros estudos (BORGES et al., 2008; MAUÉS et al., 2010; SERBIM; FIGUEIREDO, 2011; BENEDETTI; MAZO; BORGES, 2012).

Esse expressivo percentual de baixa escolaridade encontrado nos estudos evidencia uma realidade comum em países em desenvolvimento como o Brasil, pois o ensino não era uma prioridade no período em que esses idosos viveram a sua infância (HOTT; PIRES, 2011).

Em relação ao rendimento mensal, $82,8 \%$ das idosas avaliadas recebiam de dois a cinco salários mínimos, contrariando outros estudos em que o maior percentual dos idosos tem uma renda mensal em média de um a três salários mínimos. Uma possível justificativa para que os idosos pesquisados nos diferentes estudos apresentem baixa renda está no fato de a maioria deles possuírem escolaridade baixa e serem predominantemente do sexo feminino, não tendo desenvolvido atividade profissional ao longo da vida, dedicando-se às atividades domésticas (LEITE et al., 2012; TAVARES; DIAS, 2012; BRAGA et 
al., 2011; SERBIM; FIGUEIREDO, 2011). Não foi objeto de estudo investigar os possíveis motivos pela desigualdade de distribuição de renda nas diferentes regiões do país.

Em relação ao arranjo domiciliar, o presente estudo indicou que $67,2 \%$ das idosas moravam sozinhas, o que contraria resultados de outros estudos, já que a maior parte dos idosos informou morar com esposo(a), companheiro(a), parentes, empregados ou amigos (BORGES et al., 2008; HOTT; PIRES, 2011; SERBIM; FIGUEIREDO, 2011; BENEDETTI; MAZO; BORGES, 2012; LEITE et al., 2012; PONTES, 2013).

Embora não tenha sido objeto de investigação, neste estudo, analisar e avaliar o percentual de idosos que moram sozinhos, com companheiro, com familiares, com amigos ou outros, é importante ressaltar o alto índice de idosos morando sozinhos, dos quais $67,2 \%$, foram apontados no presente estudo. Por se tratar de pessoas aparentemente vulneráveis e frágeis que possam estar expostas a uma situação de risco, seja física, social ou psicológica, segundo Pontes (2013), necessitam de uma maior demanda de cuidados tanto no âmbito familiar quanto no público.

As características demográficas encontradas nesse estudo estão de acordo com outros estudos aqui citados (BORGES et al., 2008; BRAGA et al., 2011; SERBIM; FIGUEIREDO, 2011; LEITE et al., 2012). Há uma maior prevalência de mulheres em comparação ao número de homens: as mulheres têm uma maior tendência para frequentar grupos de convivência e espaços sociais, enquanto, aos homens, é mais característico ser o provedor financeiro do lar e pouco participativo desses locais (LEITE et al., 2012). Outra possível justificativa para a baixa participação masculina nos grupos de convivência pode estar relacionado ao fato de que os homens tendem a assumir novos relacionamentos e evidenciam uma maior resistência, após a aposentadoria, em participar de atividades culturais, educacionais e lúdicas (BORGES et al., 2008).

Por outro lado, a constatação de um número reduzido de participação de idosos com mais de 80 anos nos grupos estudados, pode estar associado ao fato de apresentarem maior grau de comorbidades e dependência. São fatores importantes que podem limitar o acesso e a participação dos mesmos nas atividades oferecidas nos grupos de convivência além da necessidade de auxílio de outras pessoas para levá-los aos locais dos encontros (HOTT; PIRES, 2011).

Analisando-se os resultados do WHOQOL-OLD (Tabela 2), que avalia a qualidade de vida dos idosos, mais especificamente, o escore total de qualidade vida percebida pela amostra, constatou-se que foi de 60,25 pontos de um total de 100 pontos, valor aproximado ao estudo de Carvalho et al. (2010), 66,6 pontos. Quando os domínios são examinados, constata-se uma semelhança 
nos resultados entre este estudo e o que se encontra em discussão. Na Tabela 2 , constata-se que os domínios mais bem pontuados pelas idosas foram as "Atividades passadas, presentes e futuras" (71,4) e "Participação Social" (71,4). O domínio "Atividades passadas, presentes e futuras" apresenta um escore que demonstra uma aparente positividade quanto à possibilidade de continuar alcançando realizações pessoais por parte das idosas. Da mesma forma, um escore alto para o domínio "Participação social" pode estar associado à proposta dos grupos de convivência em proporcionar atividades físicas, de recreação e de maior interação entre os idosos. Este domínio está mais diretamente associado à autonomia, a qual obteve o segundo maior escore de 68,85 pontos. Este fato, talvez, possa se justificar devido à "Autonomia" estar diretamente relacionada com a capacidade de escolha e participação social, sendo esta um dos determinantes básicos para uma boa qualidade de vida (CARVALHO et al., 2010).

O domínio que recebeu uma pontuação menor foi o relacionado à "Intimidade" $^{\prime \prime}(23,81)$, o que demonstra certa insatisfação com suas relações pessoais e íntimas e talvez esteja relacionado ao fato de a maioria das idosas estarem viúvas e residirem sozinhas. Este domínio trata de assuntos relativos a companheirismo, capacidade de amar e sexualidade. Quanto ao domínio "Morte e morrer", relacionado a valores espirituais, religião, percepção que sentem no final da vida e os temores acerca da morte, este apresentou um escore intermediário $(65,73)$. Da mesma forma, o domínio "Funcionamento dos sentidos", cujo escore apontado foi de 60,23 pontos, estando mais relacionado aos aspectos referentes ao prejuízo no funcionamento do sistema sensorial, o que indica um impacto desfavorável na qualidade de vida da amostra. Carvalho et al. (2010) acusou uma percepção melhor de qualidade de vida nos domínios "Funcionamento dos sentidos $(72,0)$ ", “Atividades passadas, presentes e futuras (68,5)" e "Intimidade" (68,1).

Considerando-se que, de um total de 100 pontos, a média dos domínios foi de 60,25 pontos, constata-se que a amostra apresenta uma qualidade de vida regular, possivelmente pelo impacto desfavorável que o idoso sofre devido ao grande número de mudanças sob seu bem-estar, principalmente na faixa etária estudada, acima de 80 anos. Achados semelhantes foram verificados no estudo de Tavares e Dias (2012). Em outros estudos, nos quais os resultados apresentaram uma percepção melhor da qualidade de vida pelos idosos, a faixa etária estudada era de 60 anos ou mais (MIRANDA; BANHATO, 2008; BRAGA et al., 2011; LIMA; BITTAR, 2012).

Serbim e Figueiredo (2011) investigaram 15 idosos sem definição de idade e, na sua maioria, não alfabetizados e verificaram que os domínios que mais contribuíram para a qualidade de vida dos avaliados foram: "Funcionamento dos sentidos" (14,8 pontos) e "Morte e morrer" (14,7 pontos), seguida da "Participação 
social" (14,4 pontos), "Autonomia" (13,9 pontos), "Intimidade" (13,0 pontos) e, com pior escore, foi "Atividades passadas, presentes e futuras" (12,8 pontos). Nos resultados do estudo de Tavares e Dias (2012), os domínios que mais contribuíram, também, foram: "Funcionamento dos sentidos" (79,95 pontos) e "Morte e morrer" (76,30 pontos), porém, a que teve pior escore foi "Autonomia" (60,44). Para Pontes (2013), o maior escore foi na faceta "Intimidade" (63,93 pontos) e a que apresentou o escore mais baixo foi "Morte e Morrer" (29,00 pontos).

Os escores apontados nos estudos citados anteriormente podem ter sido ocasionados, em consequência, de "Funcionamento dos sentidos" estar associado à perda das habilidades sensoriais nas atividades da vida diária e da capacidade de interação com outras pessoas; “Morte e morrer" estar relacionado às expectativas e temores sobre a morte; "Participação social" estar relacionado à participação em atividades do quotidiano; "Autonomia" estar relacionado à independência na velhice; "Intimidade" estar relacionado à capacidade de ter relações pessoais e intimas; e "Atividades passadas, presentes e futuras" estarem relacionadas à satisfação sobre conquistas na vida e projetos futuros.

No estudo de Maués et al. (2010), em que os idosos foram divididos em dois grupos, G1 (60-70 anos) e G2 (85 anos ou mais), não foi identificada diferença quanto ao escore total do WHOQOL-OLD, demonstrando que a idade avançada parece não promover piora da qualidade de vida. Os domínios que mais contribuíram nos dois grupos foram "Intimidade" (15,00 pontos) e "Atividades Passadas, Presentes e Futuras" (15,00 pontos), mostrando que a oportunidade de amar e sentir-se amado e de estar satisfeito com suas realizações, objetivos alcançados e projetos durante a vida, tem importante influência sobre a qualidade de vida de idosos, independentemente da faixa etária.

Entretanto, houve diferença entre os dois grupos nos domínios "Participação Social", "Autonomia" e "Morte e Morrer": respectivamente,13,00 e 14,00; 14,00 e 13,00; 16,00 e 15,00. Para os idosos jovens, a participação em atividades do cotidiano, especialmente na comunidade, e a capacidade preservada de tomar decisões, tiveram maior impacto na qualidade de vida, provavelmente pela sensação de "ser útil" para a sociedade e de "ser respeitado" e "ser ouvido" nas suas opiniões. Para o G2, o domínio com maior escore foi "Morte e Morrer" $(15,00)$, possivelmente, por estar associado à aceitação da própria finitude nesta fase da vida que pode trazer paz interior, contribuindo para viver com boa qualidade (MAUÉS et al., 2010).

No estudo de Miranda e Banhato (2008), em que se investigou os possíveis efeitos da participação de idosos em grupos de convivência na sua qualidade de vida, foi apontado que aqueles que participavam ativamente percebiam possuir melhor qualidade de vida quando comparados com aqueles, também 
ativos, porém que não participavam de nenhum grupo regularmente. Os resultados demonstraram que o domínio com maior escore foi "Funcionamento dos Sentidos", seguido da "Participação Social", e o que apresentou o escore mais baixo foi "Autonomia". No entanto, os estudos de Lima e Bittar (2012), objetivaram comparar a percepção de qualidade de vida global de idosos de ambos os sexos que participavam de um grupo, em que eram realizadas atividades de promoção da saúde com idosos que não participavam deste grupo, sendo constatado que o grupo participante dos encontros da terceira idade obteve melhores resultados relacionados à percepção de qualidade de vida global e em diversos domínios quando comparados ao grupo não participante. Os achados apontaram que os maiores escores foram para os seguintes domínios: "Funcionamento dos Sentidos", "Autonomia" e "Participação Social".

Mesmo com escores que variavam conforme a contribuição de cada domínio na qualidade de vida dos idosos nos grupos estudados, nos resultados das amostras desses estudos, (MIRANDA; BANHATO, 2008; MAUÉS et al., 2010; BRAGA et al., 2011; SERBIM; FIGUEIREDO, 2011; LIMA; BITTAR, 2012), é possível verificar que os idosos que participavam de algum grupo de convivência percebiam ter uma melhor qualidade de vida.

A participação ativa em grupos de convivência parece interferir positivamente na avaliação do idoso em relação à sua qualidade de vida, pois, de alguma forma, contribuiu na minimização de sentimentos de solidão e abandono, proporcionando um suporte social. As atividades desenvolvidas parecem ser um fator importante que ajuda a reforçar, no idoso, o sentimento de valor pessoal e uma forma de crescimento pessoal (MIRANDA; BANHATO, 2008).

Quando analisado os escores do WHOQOL-BREF no presente estudo, constatou-se que o domínio das "Relações Sociais" (69,8 pontos) apresentou o maior escore médio, seguido pelos domínios "Meio ambiente" (68,2 pontos), "Psicológico" (66,3 pontos) e "Físico" (65,2 pontos). Quanto às questões 1 e 2, referentes à autopercepção do idoso sobre a sua qualidade de vida ( 71,5 pontos) e satisfação com a saúde (65,5 pontos), de um total de 100 pontos, os dados apontaram para um escore satisfatório de qualidade de vida. Dados semelhantes foram encontrados no estudo de Pontes (2013), em que menos de 50\% dos idosos referiram ter boa qualidade de vida e estarem satisfeitos com sua saúde.

Essa mesma ordem de contribuição dos diferentes domínios, também, foi identificada no estudo de Serbim e Figueiredo (2011); “Relações Sociais", “Meio ambiente", "Psicológico" e "Físico"; já no estudo de Tavares e Dias (2012), o maior escore foi para as "Relações Sociais" (68,82 pontos). Também, no estudo de Pontes (2013), o maior escore foi para as "Relações Sociais" (68,06 pontos), enquanto o escore mais baixo foi para o domínio "Físico" (55,83 pontos). Porém, 
no estudo de Lima e Bittar (2012) os maiores escores, respectivamente, foram nos domínios "Físico", "Psicológico", "Meio Ambiente" e "Relações Sociais". No estudo de Braga et al. (2011), os maiores escores foram para "Relações Sociais" e "Físico", e o mais baixo foi o domínio "Meio Ambiente".

Ao se analisar a contribuição de cada domínio da qualidade de vida conforme os resultados descritos na tabela 2, é possível constatar que os domínios "Relações Sociais" e "Meio Ambiente" estão integrados, pois a participação social nos grupos de convivência contribui na qualidade de vida dos sujeitos, favorecendo para um maior suporte social para a realização de suas atividade cotidianas. Também o ambiente físico em que o idoso está inserido apresenta grande influência na vida dele, auxiliando para se adaptar ao meio familiar e comunitário e oportuniza para adquirir novas informações e lazer.

O domínio psicológico pode estar integrado ao domínio físico, pois o maior número de incapacidade funcional apresenta mais risco de baixa autoestima e interfere na imagem corporal e nos sentimentos positivos. O idoso pode se sentir desmotivado para o cuidado com a saúde dado às limitações impostas pela incapacidade funcional, favorecendo os sentimentos negativos (LIMA; BITTAR; TAVARES; DIAS, 2012).

Em relação ao domínio físico, cujo escore foi o mais baixo (65,2 pontos) no presente estudo, segundo Lima e Bittar (2012), esse domínio tem uma importante relevância na qualidade de vida dos idosos, pois está associado à capacidade funcional e é um fator que, também, causa impacto na autonomia e na independência dos mesmos. É importante considerar o ambiente em que o idoso está inserido, pois o domínio ambiental também influencia na qualidade de vida destes idosos. As diversas atividades que o grupo de idosos realiza, dentre elas as de cunho social, proporcionam possíveis benefícios sobre os fatores sociais e psicológicos, os quais refletem numa melhor qualidade de vida.

Em relação ao nível de atividade física total analisada através do IPAQ adaptado a idosos, o presente estudo constatou que $100 \%$ das idosas foram classificadas como insuficientemente ativas. Analisando-se separadamente os seus domínios, contata-se que, nas atividades domésticas, o ítem "Atividade física" moderada dentro de casa ou de apartamento foi a que apresentou o maior escore.

Entretanto, no estudo de Benedetti, Mazo e Borges (2012), com 1.062 idosos de 60 a 101 anos e média de 71,9 $\pm 7,6$ anos, os maiores níveis de atividade física foram observados nos domínios relacionados às atividades domésticas e de lazer. Contudo, quando realizada a análise pelos domínios do IPAQ, a maioria dos idosos foi considerada pouco ativa em todos os domínios. Resultados semelhantes foram obtidos no estudo de Castro et al. (2010) com 775 idosas de idade entre 60 a 80 anos e média de $70 \pm 6$ anos, em que $66,7 \%$ da 
amostra foram classificadas como pouco ativas. Segundo os autores, os escores encontrados apresentam uma associação em que conforme avança a idade, menor é o escore obtido.

Os maiores escores no domínio das atividades domésticas se devem ao fato do predomínio das mulheres nos grupos de convivência. Possivelmente, outro fator que possa ter contribuído é a tendência de as idosas exercerem, ao longo de suas vidas, um papel restrito ao ambiente doméstico. A maioria dessas mulheres que hoje frequentam os grupos não desenvolveu outras atividades profissionais, mas pelo contrário: elas se dedicaram às atividades do lar e a ser mães e esposas (LEITE et al., 2012).

\section{Conclusão}

É importante conhecer o processo de envelhecimento desse contingente populacional e buscar estratégias de planejamento, implementação e avaliação dos programas de promoção de saúde e que favoreçam um envelhecimento saudável e, consequentemente, uma melhor qualidade de vida aos idosos uma vez que esta fase da vida precisa ser mais bem compreendida e respeitada em sua plenitude.

Ter qualidade de vida é uma condição de responsabilidade coletiva, pois vários fatores interferem diretamente: desde os pessoais e sociais até os culturais, econômicos e governamentais. Portanto, deve ser uma construção permanente e constante para que a sociedade esteja preparada para envelhecer com qualidade.

As idosas avaliadas apresentaram uma percepção satisfatória sobre a sua satisfação com a saúde e sua qualidade de vida, e a maior pontuação foi observada nos domínios "Atividades passadas, presentes e futuras" e "Participação social" e, a menor, no domínio "Intimidade". Quanto à autopercepção do idoso sobre a sua qualidade de vida e a satisfação com a saúde, de um total de 100 pontos, os dados apontaram para um escore de bom a regular de qualidade de vida. Salienta-se que o domínio das "Relações Sociais" apresentou o maior escore médio, seguido pelos domínios "Meio ambiente", "Psicológico" e "Físico".

No que refere à prática da atividade física, a amostra demonstrou estar insuficientemente ativa, isto é, não alcançou os índices mínimos para uma prática que beneficiasse à saúde.

Os grupos de convivência podem ser um dos caminhos para que as variáveis avaliadas possam ser mais bem exploradas, pois conseguem atingir um número significativo de idosos e propiciam aos participantes criar condições para promover sua autonomia, integração e participação efetiva na sociedade, 
além de influenciar de modo positivo no seu bem-estar psíquico e físico, contribuindo ainda para minimizar os sentimentos de solidão e abandono.

Sugere-se a realização de novas investigações nessa faixa etária a fim de completar e comparar os resultados de qualidade de vida obtidos no presente estudo.

\author{
PERCEIVED QUALITY OF LIFE \\ AND LEVEL OF PHYSICAL ACTIVITY: A STUDY \\ OF ELDERLY WOMEN ABOVE 80 \\ YEARS OF AGE PARTICIPATING IN A MUNICIPAL \\ HEALTH PROGRAM FOR SENIORS \\ IN THE SERRA GAUCHA REGION, \\ RIO GRANDE DO SUL STATE
}

abstract

This is a descriptive cross-sectional study that aimed to investigate the perceived quality of life and the physical activity level of elderly women above 80 years participating in a municipal health program for the seniors in the mountainous region of the state of Rio Grande do Sul, Brazil. Fifty-eight elderly women aged 80 years or more were evaluated. Data were collected between October and November 2013 using the following research instruments: a sociodemographic questionnaire, the World Health Organization Quality of Life (WHOQOL)-OLD questionnaire, and the International Physical Activity Questionnaire (IPAQ), adapted for the elderly. Results were tabulated and evaluated by descriptive analysis and Spearman correlation using the Statistical Package for Social Sciences (SPSS) software version 20.0. The results of the present study found that $94.8 \%$ of the women did not complete elementary school, $82.8 \%$ received from two to five minimum wages, $67.2 \%$ lived alone, and $91.4 \%$ were widows. With a mean WHOQOL-OLD score of $60.25 \pm 8.25$, the elderly women had a fair perception of their satisfaction with health and their quality of life. The highest scores in this questionnaire were observed in the domains "Past, present and future activities" (71.44 \pm 10.73 ) and "Social participation" (71.44 \pm 10.73$)$, and the lowest score was observed in the domain "Intimacy" (23.81 \pm 30.35$)$. In addition, the lowest scores in the WHOQOL-Bref questionnaire were found in the physical domain, related to pain/discomfort, energy/fatigue, sleep/rest, mobility, daily activities, dependence on medication or treatments, and/ or working capacity (65.20 \pm 9.93$)$. With regard to physical activity as evaluated by the different domains of the IPAQ, 100\% of the women 
were classified as insufficiently active. It was concluded that the elderly women showed a satisfactory perception of their satisfaction with health and quality of life and an insufficient level of physical activity.

keywords

Quality of Life. Physical Activity. Aging.

referências

AIRES, Marinês Aires; PASKULIN, Lisiane Manganelli Girardi; MORAIS, Eliane Pinheiro de. Capacidade funcional de idosos mais velhos: estudo comparativo em três regiões do Rio Grande do Sul. Revista Latino-Americana de Enfermagem, Ribeirão Preto, v. 18, n. 1, p. 11-17, fev. 2010

ALENCAR, Maria do Socorro Silva: CARVALHO, Cecília Maria Resende Gonçalves de. O envelhecimento pela ótica conceitual, sociodemográfica e político-educacional: ênfase na experiência piauiense. Interface - Comunicação, Saúde, Educação, Botucatu, v. 13, n. 29, p. 435-444, jun. 2009.

ALEXANDRE, Tiago da Silva; CORDEIRO, Renata Cereda; RAMOS, Luiz Roberto. Factors associated to quality of life in active elderly. Revista de Saúde Pública. São Paulo, v. 43, n. 4, ago. 2009

ARAÚJO, Maria Odete Pereira Hidaldo de; CEOLIM, Maria Filomena. Avaliação do grau de independência de idosos residentes em instituições de longa permanência. Revista da Escola de Enfermagem da USP, São Paulo, v. 41, n. 3, p. 378-385, 2007.

BENEDETTI, Tânia Rosane Bertoldo; MAZO, Giovanna Zarpellon; BORGES, Lucélia Justino. Condições de saúde e nível de atividade física em idosos participantes e não participantes de grupos de convivência de Florianópolis. Ciência \& Saúde Coletiva, Rio de Janeiro, v. 17, n. 8, p. 2087-2093, ago. 2012

BORGES, Paula Lutiene de Castro; BRETAS, Rose Procópio: AZEVEDO, Silvana Fernandes; BARBOSA, Juliana Magalhães Machado. Perfil dos idosos frequentadores de grupos de convivência em Belo Horizonte, Minas Gerais, Brasil. Cadernos de Saúde Pública, Rio de Janeiro, v. 24, n. 12, p. 2798-2808, dez. 2008.

BRAGA, Maria Cecília Portugal; CASELLA, Milla Apolinário: CAMPOS, Maria Laura Nogueira; PAIVA, Sabrina Pereira. Qualidade de vida medida pelo WHOQOL-BREF: estudo com idosos residentes em Juiz de Fora, MG. Revista de APS: Atenção Primária à Saúde, Juiz de Fora, v. 14, n. 1, p. 93-100, jan./mar. 2011.

CONSELHO NACIONAL DE SAÚDE (CNS). Comissões CNS - CONEP. Resolução No 251, de 7 de agosto de 1997. Disponível em: <http://conselho.saude.gov.br/ web_comissoes/conep/aquivos/resolucoes/resolucoes.htm>. Acesso em: 9 jul. 2012

Resolução № 196 de 10 de outubro de 1996. Disponível em: <http://conselho. saude.gov.br/resolucoes/reso_96.htm>. Acesso em: 9 jul. 2012.

CAXIAS DO SUL. Secretaria Municipal do Esporte e Lazer. Conselho Municipal do Idoso de Caxias do Sul. Projeto Conviver. Disponível em: <http://www.caxias.rs.gov. br/esporte_lazer/texto.php?codigo=50>. Acesso em: 10 nov. 2012

CARVALHO, Euller Duarte de: VALDARES, Ana Lúcia Ribeiro: COSTA-PAIVA, Lúcia Helena da: MORAIS, Sirlei Siani; PINTO NETO, Aarão Mendes. Atividade física e qualidade de vida em mulheres com 60 anos ou mais: fatores associados. Revista Brasileira de Ginecologia e Obstetrícia, Rio de Janeiro, v. 32, n. 9, p. 433-440, set. 2010 
DOMINGUES, Paula Casalini; NERI, Anita Liberalesso. Atividade física habitual, sintomas depressivos e doenças auto-relatadas em idosos da comunidade. Revista Brasileira de Atividade Física e Saúde, Pelotas, v. 14, n. 3, p. 164-173, set./dez. 2009.

FLECK, Marcelo P.; CHACHAMOVICH, Eduardo; TRENTINI, Clarissa. Development and validation of the Portuguese version of the WHOQOL-OLD module. Revista de Saúde Pública, São Paulo, v. 40, n. 5, p. 785-791, out. 2006.

HOTT, Alanna Magalhães; PIRES, Vitória Augusta Teles Netto. Perfil dos idosos inseridos em um centro de convivência. Revista Enfermagem Integrada, Ipatinga, v. 4, n. 1, p. 765-778, jul./ago. 2011

INSTITUTO BRASILEIRO DE GEOGRAFIA E ESTATÍSTICA (IBGE). Censo Demográfico 2010. Disponível em: <http://www.ibge.gov.br/home/estatistica/populacao/censo2010/ calendario.shtm>. Acesso em: 4 jul. 2012.

LACOURT Marcelle Xavier; MARINI Lucas Lima. Decréscimo da função muscular decorrente do envelhecimento e a influência da qualidade de vida do idoso: uma revisão de literatura. Revista Brasileira de Ciências do Envelhecimento Humano. Passo Fundo, v. 3, n. 1, p. 114-121, jan./jul. 2006.

LEITE, Marinês Tambara; HILDEBRANDT, Leila Mariza; KIRCHNER, Rosane Maria; WINCK, Marisa Teresinha; SILVA, Luiz Anildo Anacleto; FRANCO, Gianfábio Pimentel. Estado cognitivo e condiç̃es de saúde de idosos que participam de grupos de convivência. Revista Gaúcha de Enfermagem, Porto Alegre, v. 33, n. 4, p. 64-71, dez. 2012.

LIMA, Lara Carvalho Vilela; BITTAR, Cléria Maria Lobo. A percepção da qualidade de vida em idosos: um estudo exploratório. Revista Brasileira de Qualidade de Vida, Ponta Grossa, v. 4, n. 2, p. 1-11, jul./dez. 2012.

MATSUDO, Sandra Mahecha. Avaliação do idoso: física e funcional. Londrina: Midiograf, 2000

MAUÉS, Cristiane Ribeiro; PASCHOAL, Sérgio Márcio Pacheco; JALUUL, Omar; FRANCCA, Cristina Claro; FILHO. Wilson Jacob. Avaliação da qualidade de vida: comparação entre idosos jovens e muito idosos. Revista da Sociedade Brasileira de Clínica Médica, São Paulo, v. 8, n. 5, p. 405-410, set./out. 2010

MAZO, Giovana Zarpellon; BENEDETTI, Tania Rosane Bertoldo. Atividade física e o idoso: concepção gerontológica. 3. ed. Porto Alegre: Sulina, 2009.

MAZO, Giovana Zarpellon; BENEDETTI, Tania Rosane Bertoldo. Adaptação do questionário internacional de atividade física para idosos. Revista Brasileira de Cineantropometria e Desempenho Humano, Florianópolis, v. 12, n. 6, p. 480-484, jun. 2010.

MIRANDA, Luciene Corrêa; BANHATO, Eliane Ferreira Carvalho. Qualidade de vida na terceira idade: a influência da participação em grupos. Psicologia em Pesquisa, Juiz de Fora, v. 2, n. 1, p. 69-80, jan./jun. 2008.

OLIVEIRA, Aldalan Cunha de; OLIVEIRA, Núcia Macêdo Diniz; ARANTES, Paula Maria Machado; ALENCAR, Mariana Asmar. Qualidade de vida em idosos que praticam atividade física - uma revisão sistemática. Revista Brasileira de Geriatria e Gerontologia, Rio de Janeiro, v. 13, n. 2, p. 301-312, ago. 2010

PONTES, Maria de Lourdes de Farias. Qualidade de vida e fragilidade em idosos que residem em comunidades. 2013. 175 f. Dissertação (Mestrado) - Escola de Enfermagem de Ribeirão Preto, Universidade de São Paulo, Ribeirão Preto, 2013.

SANTOS, Kelly Antunes dos; KOSZUOSKY, Ricardo: DIAS-DA-COSTA Juvenal Soares; PATTUSSI, Marcos Pascoal. Fatores associados com a incapacidade funcional em idosos do Município de Guatambu, Santa Catarina, Brasil. Cadernos de Saúde Pública, Rio de Janeiro, v. 23, n. 11, p. 2781-2788, nov. 2007. 
SERBIM, Andreivna Kharenine; FIGUEIREDO, Ana Elizabeth Prado Lima. Qualidade de vida de idosos em um grupo de convivência. Scientia Medica, Porto Alegre, v. 21 n. 4, p. 166-172, out./dez. 2011

TAVARES, Darlene Mara dos Santos; DIAS, Flavia Aparecida. Capacidade funcional, morbidades e qualidade de vida de idosos. Texto \& Contexto Enfermagem, Florianópolis, v. 21, n. 1, p. 112-120, jan./mar. 2012

XAVIER, André Junqueira: D'ORSI, Eleonora: SIGULEM, Daniel: RAMOS, Luiz Roberto. Orientação temporal e funções executivas na predição de mortalidade entre idosos: estudo Epidoso. Revista de Saúde Pública, São Paulo, v. 44, n. 1, p. 148-158, fev. 2010

Recebido: 18/07/2014

Aceite Final: 10/12/2014 